\title{
On the determination of the granular size of the economy
}

\author{
Omar Blanco-Arroyo Alba Ruiz-Buforn David Vidal-Tomás \\ Simone Alfarano
}

June 26, 2018

\section{Highlights}

- A method to estimate the granular size of an economy is proposed.

- The Spanish economy is granular.

- The granular size of the Spanish economy is composed by the 450 largest firms.

\begin{abstract}
Introducing the granular hypothesis, Gabaix (2011) shows that the idiosyncratic shocks of a few "granular" firms account for a significant fraction of aggregate fluctuations of the US business cycle. In the literature, however, the question of how many are the granular firms in an economy is left unanswered. Using Spanish data, we propose a novel methodology to calibrate the granular size of the economy, i.e. the number of granular firms.
\end{abstract}

\section{Introduction}

In mainstream macroeconomics, firm-level idiosyncratic shocks are assumed to average out in aggregate (Lucas, 1977), contributing just marginally to macroeconomic fluctuations. This idea has been challenged by the empirical work of Gabaix (2011), who explicitly tests on what extent those shocks account for aggregate fluctuations. He has shown that the idiosyncratic shocks to the largest 100 firms have a significant impact on the business cycle fluctuations of United States, accounting approximately for one-third of GDP variations. Aggregate fluctuations, therefore, can be partially attributed to the destinies of well identified "grains", which are few very large firms. If 
an economy is characterised by such behaviour, it is defined as a granular economy.

After the seminal work of Gabaix (2011), other studies have found that several macroeconomic variables exhibit granular fluctuations, such as exports (del Rosal, 2013; di Giovanni et al., 2017) or investments (Grullon et al., 2013). The granular behaviour can also be observed at sectoral level, for example in the banking (Blank et al., 2009) or manufacturing sector (Wagner, 2012). In those empirical contributions, however, the number of what are considered granular firms is exogenously given.

Based on the methodology proposed by Gabaix (2011), in this paper, we aim at calibrating how many are the granular firms, i.e. to determine the granular size of the economy. To the best of our knowledge, we are the first to address this issue in the literature.

The paper is organised as follows: the description of the data and the empirical methodology is presented in Section 2. Section 3 shows the main results and Section 4 concludes.

\section{Data and Methodology}

In order to perform our empirical analysis, we use the SABI (Sistema de Análisis de Balances Ibéricos) database, which collects accounting data from Spanish firms. For our purpose, we are interested in the annual volume of sales and the corresponding number of employees as well as the activity carried out by each individual firm, which is coded in the SIC code. The initial sample obtained from SABI is made up of the 10000 largest Spanish firms ${ }^{1}$ in the period ranging from 1995 to 2016, ranked by their volume of sales. Following Gabaix (2011), firms whose SIC codes are among the following numbers have been filtered out: 1311, 1389, 2911, 2999, between 4900 and 4940, 5052, 5172 and between 6000 and 6999. These firms are, in fact, engaged in activities whose impact on their sale fluctuations are directly related to changes in world commodity prices (e.g. oil companies), which cannot be considered idiosyncratic shocks, or are financial companies, whose sales do not stem from manufactured goods (e.g. banks). After the filtering procedure, the number of remaining firms is 9072. Macroeconomic data (GDP, GDP per capita and GDP deflator) are taken from the World Bank's Development Indicators database.

\footnotetext{
${ }^{1}$ The sum of their sales accounts for approximately $70 \%$ of GDP of the Spanish economy.
} 
As proposed by Gabaix (2011), we construct the measure of the idiosyncratic labour productivity shocks to the top $K$ firms, which is called granular residual:

$$
\Gamma_{t}=\sum_{i=1}^{K} \frac{S_{i, t-1}}{Y_{t-1}}\left(g_{i, t}-\bar{g}_{t}^{Q}\right),
$$

where $S_{i, t-1}$ is the deflated volume of sales of firm $i$ in year $t-1, Y_{t-1}$ is the real GDP in year $t-1$ and $g_{i, t}-\bar{g}_{t}^{Q}$ is the demeaned labour productivity growth rate, considered as a proxy for the idiosyncratic shock to firm $i$ in year $t$. The term $\bar{g}_{t}^{Q}$ is the cross-sectional median of $g_{i, t}$ computed among the top $Q$ firms, with $Q \geq K$. Labour productivity growth of firm $i$ in year $t$ is defined as:

$$
g_{i, t}:=\Delta \ln \left(\frac{S_{i, t}}{E_{i, t}}\right)=\ln \left(\frac{S_{i, t}}{E_{i, t}}\right)-\ln \left(\frac{S_{i, t-1}}{E_{i, t-1}}\right),
$$

where $E_{i, t}$ is the number of employees of firm $i$ in year $t$. In order to avoid the effect of outliers, the demeaned productivity growth rates have been winsorized at $90 \%$ level.

Following Gabaix, we employ the explanatory power $\left(R^{2}\right)$ of the following regression to assess to which extent idiosyncratic shocks account for aggregate fluctuations:

$$
g_{t}^{Y}=\alpha+\sum_{i=0}^{2} \beta_{i} \Gamma_{t-i}+\varepsilon_{t},
$$

where $g_{t}^{Y}$ is per capita real GDP growth rate. Based on the Hulten's theorem (Hulten, 1978), Gabaix (2011) illustrates how the coefficients $\beta_{i}$ s provide an estimation of the factor usage. ${ }^{2}$ In order to have an intuition for the value of the factor usage of the Spanish economy, we apply the approximate calculation proposed by Gabaix (2011):

$$
\sigma_{G D P}=\mu \cdot \sigma_{\pi} \cdot h
$$

where $h=0.048$ is the square root of the Herfindahl index for sales of the 100 largest firms, $\sigma_{\pi}=0.13$ is their cross-sectional standard deviation of the productivity growth rate, averaged across the entire period, and $\sigma_{G D P}=$ 0.024 is the estimated GDP standard deviation in the considered period.

\footnotetext{
${ }^{2}$ The estimation of the factor usage provided by $\beta_{i}$ is a combination of the elasticity of substitution of labor and output elasticities with respect to production inputs.
} 
From eq. (4), the calibrated value of the factor usage is $\mu=3.8$. The estimated coefficients from equations (3) and (4) exhibit, indeed, similar values (see Table 1 ).

\section{Results}

\subsection{The Spanish economy is granular}

We first check whether the Spanish economy is granular by computing the explanatory power of the granular residual for a given number of large firms. Table 1 shows the results of the estimation of the coefficients $\beta_{i}$ s, considering different specifications of the OLS in eq. (3). With $K=Q=100$, our results $^{3}$ indicate that the Spanish economy is granular since the granular residual accounts approximately for $45 \%$ of variations of GDP growth. This value turns out to be higher than the explanatory power reported by Gabaix for the American economy. Our results provide a further empirical support to the granular hypothesis, extending its validity to the Spanish economy.

The identification of the Spanish (or American) economy as a granular economy is based on an exogenous choice for the number of large firms in eq. (1). Such "pointwise" estimation of the $R^{2}$ does not provide information on the extent of the granular region since the number of considered firms is arbitrarily chosen. Therefore, we may underestimate the contribution of the granular term to the GDP fluctuations, considering too few granular firms, or overestimate its impact, including too many firms in eq. (3).

\subsection{The granular size of the Spanish economy}

We propose a novel methodology in order to calibrate the granular size of the economy, using the Spanish data as an illustrative example. To be more precise, our aim is to calibrate the number of the granular firms, $K^{*}$. As a first step, we analyse how the explanatory power of the granular residual behaves when we progressively increase $K$ in eq. 1 , in the range $1 \leq K \leq$ $Q=1000 .{ }^{4}$ Figure 1 shows the evolution of the $R^{2}$ as a function of $K$, to which we refer as the "granular curve" (the upper curve in Figure 1). This curve is characterised by: (i) a sharp increase of the $R^{2}$ when a reduced

\footnotetext{
${ }^{3}$ We analyse the specification $K=Q=100$ to have a direct comparison to the estimates reported by Gabaix (2011).

${ }^{4}$ We now include one lag in the OLS because of the short length of the time series. Our results are robust when including two lags or considering the entire sample of available firms (material upon request).
} 
Table 1: Results of the regression of eq. (3) when $Q=K=100$.

\section{GDP Growth}

(1)

\begin{tabular}{lccc}
\hline$\Gamma_{t}$ & $\begin{array}{c}2.52^{* *} \\
(1.24)\end{array}$ & $\begin{array}{c}1.84^{*} \\
(1.02)\end{array}$ & $\begin{array}{c}2.14^{* *} \\
(0.94)\end{array}$ \\
& & $3.06^{* * *}$ & $2.45^{* *}$ \\
$\Gamma_{t-1}$ & $(0.95)$ & $(0.93)$ \\
& & & \\
$\Gamma_{t-2}$ & & & $2.19^{* * *}$ \\
& & & $(0.61)$ \\
Intercept & $0.0187^{* * *}$ & $0.0233^{* * *}$ & $0.0270^{* * *}$ \\
& $(0.0053)$ & $(0.0051)$ & $(0.0048)$ \\
\hline $\mathrm{N}$ & & & \\
$\mathrm{R}^{2}$ & 22 & 21 & 20 \\
Adj. $\mathrm{R}^{2}$ & 0.185 & 0.421 & 0.537 \\
\hline & 0.144 & 0.357 & 0.451 \\
\hline
\end{tabular}

Per capita GDP growth $g_{t}^{Y}$ is regressed on the granular residual $\Gamma_{t}$ in column 1, adding one lag in column 2 and adding two lags in column 3. Robust standard errors to autocorrelation are given in parentheses. ${ }^{* * *}$ Significance level at the $1 \%,{ }^{* *} 5 \%,{ }^{*} 10 \%$.

number of large firms is gradually included in the calculation of the granular residual (roughly the largest one hundred firms); (ii) an almost steady value of the $R^{2}$ when including additional firms.

In order to validate our results, let us introduce the equal-weight benchmark by replacing the empirical weights in eq. (1) with constant weights for all firms, i.e. posing $S_{i t}=S_{t}^{*}$, while keeping unchanged the corresponding idiosyncratic shocks. ${ }^{5,6}$ Such benchmark quantifies the contribution of the

\footnotetext{
${ }^{5}$ We consider the volume of sales $S_{t}^{*}=S_{1000, t}$ of the largest 1000th firm for each year $t$, and we assign its value to all firms in that year when computing $\Gamma_{t}$. The choice of the particular value for $S_{t}^{*}$ is irrelevant for the behaviour of the benchmark, as soon as $S_{t}^{*}$ does not coincide with the size of a granular firm.

${ }^{6}$ We limit the variability of $\beta_{i}$ to the interval $[0,3.5]$ in order to avoid that the coefficients $\beta_{i}$ in the regression (3) increase artificially their value. The upper bound is chosen as a conservative value, averaging the estimated coefficients from Table 1 and the calibrated
} 


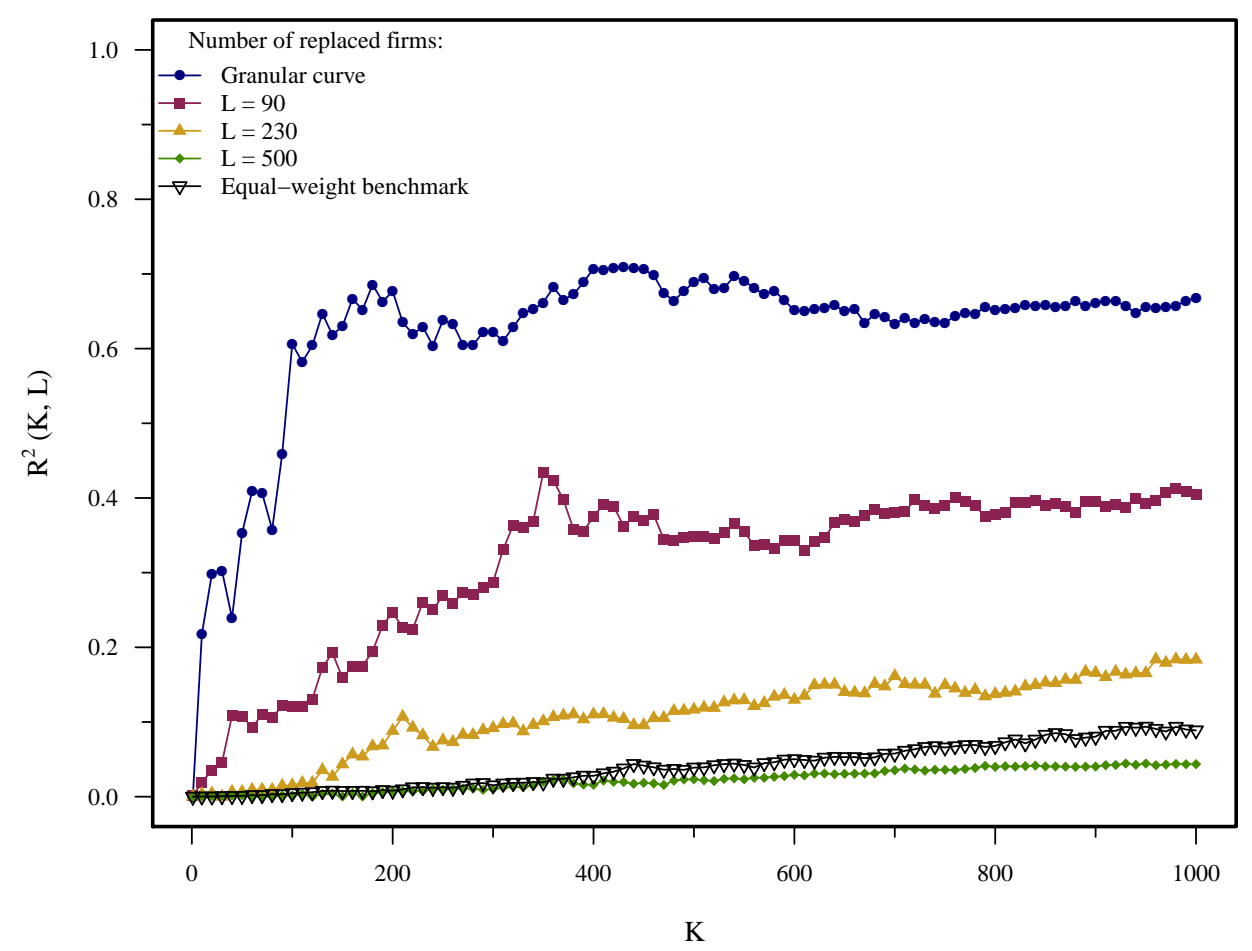

Figure 1: Explanatory power of the regression in eq. (3) as a function of an increasing number of firms $K$ and for different values of $L, R^{2}(K, L)$. The incremental step is $\Delta K=10$.

granular residual to the GDP fluctuations of an economy composed by equalsize firms (representative firm). Within the representative firm framework, the contribution of the firm-level idiosyncratic shocks to aggregate fluctuations is, indeed, marginal. The comparison of the equal-weight benchmark to the granular curve gives a clear indication of the relevant role played by the very large firms in the characterisation of business cycle fluctuations. Our results indicate that the heterogeneity of firms cannot be discarded

value of $\mu$ from eq. (4). Without introducing the bounded interval for $\beta_{i}$, the coefficients can exhibit values unrealistically high (some time higher than 30 ), considering that $\beta_{i}$ are proxies for the factor usage. Interestingly, when computing the granular curve, the coefficients $\beta_{i}$ never crosses the boundaries. 


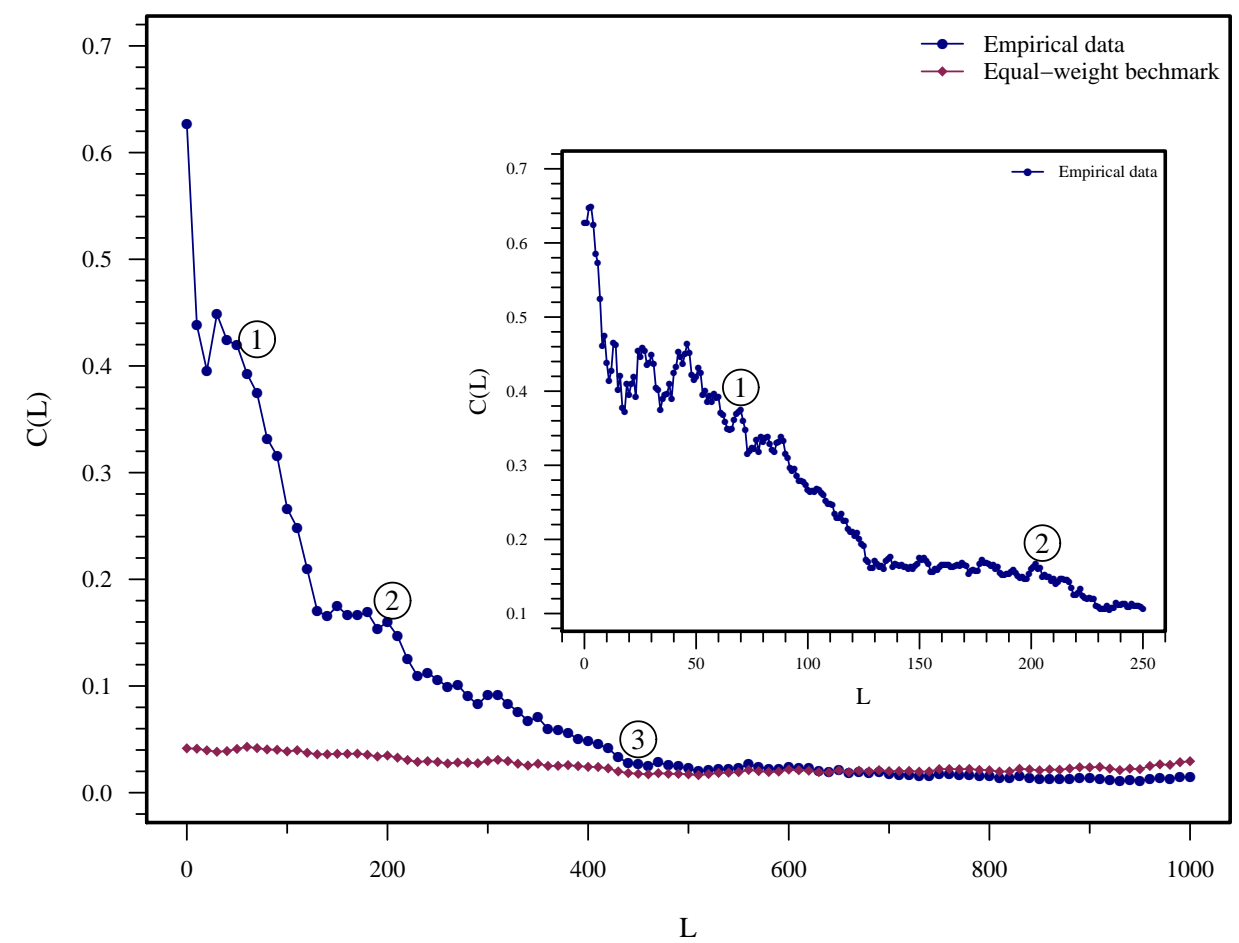

Figure 2: Evolution of the $C(L)$ curve and the equal-weight benchmark.

when modelling aggregate fluctuations. As a further evidence of the importance of the heterogeneity of firms, Figure 1 shows the transition from the granular curve to the equal-weight benchmark, when we progressively remove the $L$ largest firms in $\Gamma_{t}{ }^{7}$ The curves representing the explanatory power of the OLS regression as a function of $K$ and for given values of $L$, $R^{2}(K, L)$, exhibit smoother curvatures for large values of $L$, reaching lower explanatory power. In particular, the curve $R^{2}(K, 500)$ is almost indistinguishable from the equal-weight benchmark, indicating that the remaining heterogeneity among firms has a negligible impact on aggregate fluctuations.

\footnotetext{
${ }^{7}$ We replace the $L$ largest firms with smaller size firms, ranging from the position $Q+1$ to $Q+L$ in the ranked sample. In this way, the considered sample is always composed of $Q$ firms.
} 
In order to calibrate the granular size of the economy, we empirically analyse the sensitivity of the $R^{2}(K, L)$ curves to increased values of $L$, i.e. to a gradual elimination of the larger firms. Figure 2 plots the average cumulative explanatory power, i.e. the average cumulative $R^{2} \mathrm{~s}$, as a function of $L$ :

$$
C(L)=\frac{1}{Q} \sum_{K=1}^{Q} R^{2}(K, L) .
$$

A simple method to calibrate $K^{*}$ is, therefore, to approximatively identify the interval where the $C(L)$ curve intersects the curve of the average cumulative explanatory power of the equal-weight benchmark. Point 3 in Figure 2 indicates that the granular size of the Spanish economy is approximately $K^{*} \approx 450$ firms.

Interestingly, the inset of Figure 2 shows that the $C(L)$ curve does not decreases steadily. Instead, it exhibits some well-defined regions where it remains almost unchanged (the plateaus indicated by points 1 and 2 in Figure 2). It seems that, within the group of granular firms, we can identify an inner granular structure, due to different degrees of heterogeneity among the granular firms. In principle, we could introduce alternative criteria to calibrate $K^{*}$, taking into account the granular inner structure. However, this comes at a cost of introducing a certain arbitrariness into the choice of $K^{*}$.

\section{Conclusions}

In this paper, we have introduced a novel methodology to calibrate the number of granular firms in an economy. We have applied such method to the Spanish economy, calibrating in approximately 450 its number of the granular firms. We plan to apply our methodology to other countries. An international comparison will allow to refine the definition of the granular size of the economy introduced in this paper, by including the empirically identified inner granular structure.

\section{Acknowledgement}

The authors are grateful for funding the Universitat Jaume I under the project P11B2015-63 and the Spanish Ministry Science and Technology 
under the project ECO2015-68469-R. David Vidal-Tomás and Alba RuizBuforn acknowledge financial support of Spanish Ministry of Education, FPU2015/01434 and FPU2014/01104, respectively.

\section{References}

Blank, S., Buch, C. M. and Neugebauer, K. (2009), 'Shocks at large banks and banking sector distress: The banking granular residual', Journal of Financial Stability 5(4), 353-373.

del Rosal, I. (2013), 'The granular hypothesis in eu country exports', Economics Letters 120(3), 433-436.

di Giovanni, J., Levchenko, A. A. and Mejean, I. (2017), 'Large firms and international business cycle comovement', American Economic Review $\mathbf{1 0 7}(5), 598-602$.

Gabaix, X. (2011), 'The granular origins of aggregate fluctuations', Econometrica 79, 733-772.

Grullon, G., Hund, J. and Weston, J. (2013), 'A granular analysis of corporate investment', available at SSRN: http://dx.doi.org/10.2139/ ssrn. 2305349.

Hulten, C. R. (1978), 'Growth accounting with intermediate inputs', The Review of Economic Studies 45(3), 511-518.

Lucas, R. E. (1977), Understanding business cycles, in 'Carnegie-Rochester conference series on public policy', Vol. 5, North-Holland, pp. 7-29.

Wagner, J. (2012), 'The German manufacturing sector is a granular economy', Applied Economics Letters 19(17), 1663-1665. 